\title{
Designing a freeform optic for oblique illumination
}

Ross D. Uthoff, Rachel N. Ulanch, Kaitlyn E. Williams, Liliana Ruiz Diaz, Page King, et al.

Ross D. Uthoff, Rachel N. Ulanch, Kaitlyn E. Williams, Liliana Ruiz Diaz, Page King, R. John Koshel, "Designing a freeform optic for oblique illumination," Proc. SPIE 10590, International Optical Design Conference 2017, 105900X (27 November 2017); doi: 10.1117/12.2296876

SDIE Event: International Optical Design Conference - IODC 2017, 2017, Denver, United States 


\title{
Designing a Freeform Optic for Oblique Illumination
}

\author{
Ross D. Uthoff*, Rachel N. Ulanch, Kaitlyn E. Williams, Liliana Ruiz \\ Diaz, Page King, and R. John Koshel \\ College of Optical Sciences, The University of Arizona, 1630 University Blvd., Tucson, AZ 85721 \\ *Corresponding author: rossuthoff@email.arizona.edu
}

\begin{abstract}
The Functional Freeform Fitting $\left(\mathrm{F}^{4}\right)$ method is utilized to design a freeform optic for oblique illumination of Mark Rothko's Green on Blue (1956). Shown are preliminary results from an iterative freeform design process; from problem definition and specification development to surface fit, ray tracing results, and optimization. This method is applicable to both point and extended sources of various geometries.
\end{abstract}

OCIS codes: (089.2740) Geometric optical design, (220.2945) Illumination design , (080.4298) Nonimaging optics, (080.4225) Nonspherical lens design

\section{Introduction}

While typically not the focus of a museum visit, lighting of the objects is a critical issue to museum directors and curators and affects the visitor experience. In the case of paintings, colors can be enhanced or subdued by the source's correlated color temperature $(\mathrm{CCT})$ or color rendering index (CRI). Certain sections of the artwork can be unintentionally highlighted by non-uniform lighting or visually ruined altogether by glare. Ultimately, all illumination is damaging with higher energy ultraviolet (UV) wavelengths being the most problematic.

Improper illumination leads to faded pigments and decreased value. As prices for single works reach upwards of $\$ 300 \mathrm{M}$ (and priceless cultural value), maintaining the integrity and value of these masterworks is of utmost importance [1]. Despite its importance, most museum lighting designers are constrained to using pre-existing infrastructure for track lighting and luminaires emitting a circular light pattern. The track lighting puts the artwork under highly oblique illumination causing an illuminance gradient from the top of the artwork to the bottom and an elliptical light distribution from the previously circular luminaire output.

However, most artwork is not elliptical in shape and artists likely do not want all of the observer's attention to be drawn to the top of the painting. Achieving the desired uniformity and irradiance distribution in oblique illumination, such as a wall wash, presents a difficult optical design problem due to the variation in optical path lengths and multiple cosine dependencies.

In collaboration with The University of Arizona Museum of Art (UAMA), a custom illumination solution for the museum's most renowned painting, Mark Rothko's Green on Blue (1956, Figure 1) is developed to provide a uniform, rectangular light pattern to the painting wall with no light spill onto the side walls, floor, or wall above the painting.

\section{Lighting a Rothko}

The geometry of the problem (Figure 2) with a large cosine to the fourth falloff from the top of the painting wall to the bottom, and the extended nature of the source requires the use of a freeform optic. Freeform optical design is a technique that allows designers to push the boundaries of optical design and system performance. Typical design methods utilize analytic methods to build a surface profile point by point [3-6]. Until recently, direct design methods were limited to point sources and resulted in a point cloud surface representation $[7,8]$. These methods are often constrained by application, source emission pattern, rotational symmetry, and source size. The $(x, y, z$,weight $)$ parameterization of the point cloud prohibits refinement through optimization due to the sheer number of variables if appropriate sampling is used.

The Functional Freeform Fitting $\left(\mathrm{F}^{4}\right)$ method parameterizes the freeform surface with the coefficients of an orthonormal polynomial function. This limited number of variables leads to efficient optimization that can 


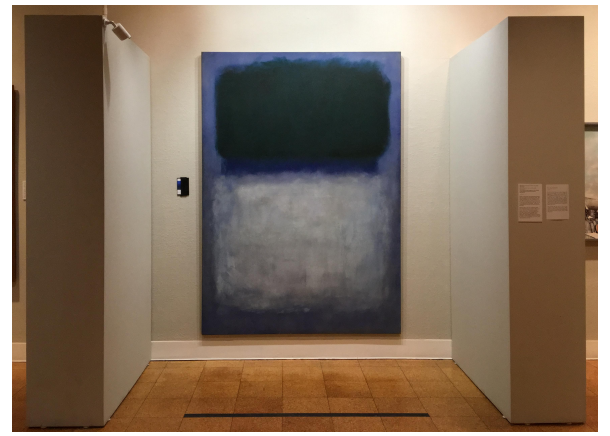

Fig. 1: Mark Rothko's Green on Blue (1956) as installed at UAMA

\begin{tabular}{lrl} 
Parameter & Value & Units \\
\hline Illuminance & $15-50$ & $\mathrm{lux}$ \\
CCT & 3000 & $\mathrm{~K}$ \\
CRI & 85 & \\
Illumination variation & $<25 \%$ & \\
Illumination area & $2464 \times 2794$ & $\mathrm{~mm}$ \\
Pointing angle & -62.7 & degrees
\end{tabular}

Table 1: Illumination system design criteria

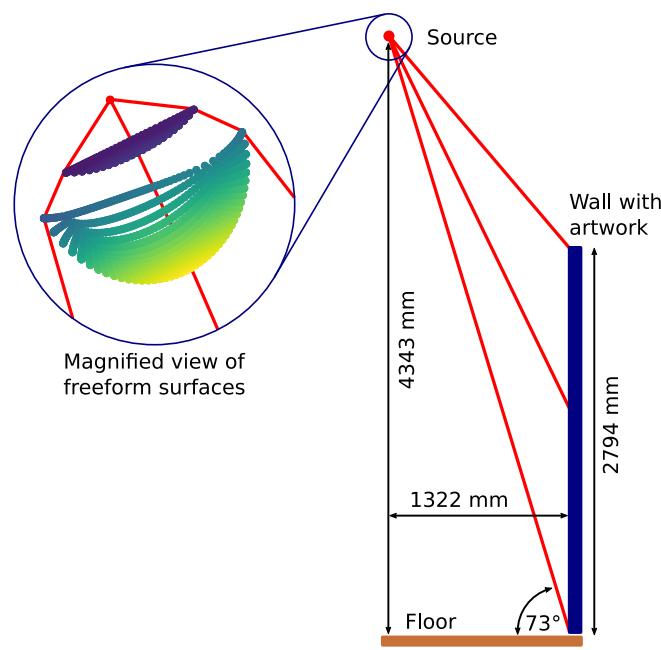

Fig. 2: $y z$-axis view of the illumination system geometry for Mark Rothko's Green on Blue (1956)

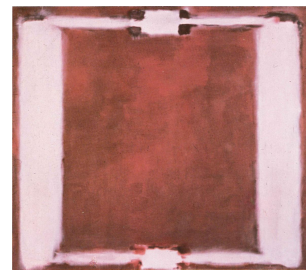

(a) Panel Five, original (1963)

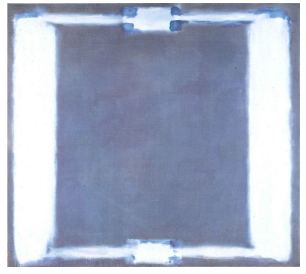

(b) Panel Five, faded (1988)
Fig. 3: Mark Rothko's Harvard mural Panel Five (1963) showing the fading over time due to incorrect illumination [2]

flexibly handle various source characteristics (radiance, size), various design geometries, and in the future, system assembly tolerances.

The source requirements for illuminating a Rothko are very stringent. The materials used by Rothko (including the use of whole eggs as a binder [9]) have a propensity for fading as evidenced by his Harvard murals (Figure 3) $[2,9,10]$. 'High responsivity' works of this kind need a visible spectrum lux limit of 50 lux with no UV content to minimize damage $[11,12]$.

In an unpublished essay by Christopher Rothko, Mark Rothko's son, he recounts how Mark would visit the first permanent Rothko exhibit at The Philips Collection in Washington, DC, and turn down the lights every time [13]. Christopher writes,

In truth, finding the optimal light level is a purely empirical process. Begin with the dimmer all the way down. Increase the light slowly, watching the colors enliven. Continue increasing the amount of light until you notice a white, almost powdery haze begin to form on the surface. Then decrease by ten percent.

Rothko was particular about how his work was to be displayed. He wanted the observer to be immersed in the work; to see the painting the same way Rothko had while creating it [14]. Experiments at UAMA with Green on Blue found the light level that brings out the work's 'glow' to be about 15 lux, allowing both proper presentation and preservation. 


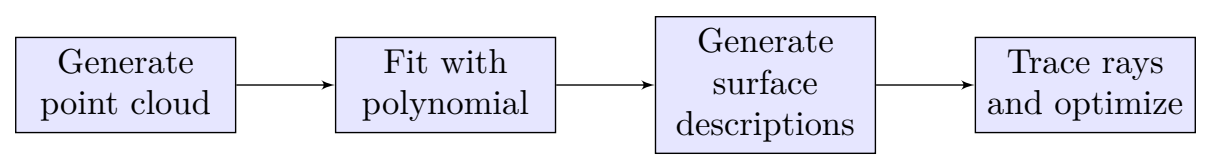

Fig. 4: Flowchart of freeform optic creation, fitting, and ray tracing

Typical museum lighting utilizes halogen lamps with $\sim 70 \mu \mathrm{W} /$ lumen of UV output $[11,12]$. As solid state lighting proliferates in museum settings [15-17], the light emitting diode (LED) provides a low cost, high luminous efficacy, energy efficient, limited UV output source with correlated color temperature flexibility.

The design parameters are provided in Table 1. The illuminance value is derived from the CIE limit and preferences of the artist. Correlated color temperature preferences for the illumination of Green on Blue were collected from UAMA staff and visitors and match the typical temperature of a halogen source $[18,19]$. The CRI follows guidelines from museum lighting experts and the IESNA [11,15]. Based on the logarithmic response of the human visual system (Fechner's Law [20]), a $<0.25$ illuminance variation as calculated by

$$
\text { coefficient of variation }=c_{\mathrm{v}}=\frac{\sigma}{\mu}
$$

is perceptually uniform. The size of the wall being illuminated and the pointing angle are derived from the geometry of the track lighting infrastructure and the painting as installed at UAMA (Figures 1 and 2). The pointing angle is calculated to point at the centerline of the painting.

\section{Method}

The Functional Freeform Fitting method follows the flowchart shown in Figure 4.

\subsection{Generate point cloud}

A point cloud is generated using a Lambertian point source-based refractive freeform lens design method [3, $4,7,8]$. The LightTools 8.5 Freeform Designer (Synopsys, Mountain View, CA) module was utilized to iterate through multiple design forms. Additionally, the module allows the addition of a Cartesian oval surface to increase the collection angle of the point source. The design forms were downselected for further evaluation based on (i) thickness greater than $10 \mathrm{~mm}$ but less than $20 \mathrm{~mm}$, (ii) efficiency greater than $90 \%$, (iii) point source collection angle greater than $50^{\circ}$, (iv) a Cartesian oval radius greater than $8 \mathrm{~mm}$ (for better acceptance of an extended source), (v) the freeform surface and Cartesian oval do not intersect, and (vi) an aspect ratio suitable for manufacturing. The selected design has a $15 \mathrm{~mm}$ thickness and collects $\pm 58^{\circ}$ of the point source. A 2D view of the freeform point cloud and Cartesian oval is shown in Figure 2.

\subsection{Polynomial fitting}

Orthonormal polynomial functions are preferred for fitting and optimization [21]. The point cloud is not rotationally symmetric, requiring a Cartesian basis. Initially, Legendre and Zernike polynomials are explored with the fitting functions built using recursive forms up to the desired order.

Zernike polynomials are a polar basis [22] and need conversion. Cartesian Zernike polynomials are built using the generating function [23]

$$
f(x, y)=\sum_{n=0}^{\infty}\left[\left(\sum_{m=1}^{n} \sum_{k=0}^{1} a_{j} Z_{n}^{(-1)^{k} m}(x, y)\right)+a_{(n+1)^{2}} Z_{n}^{0}(x, y)\right]
$$

where the $j^{\text {th }}$ coefficient is

$$
j=(n+1)^{2}-2 m+k .
$$

Recursive Cartesian forms are developed from the polar forms

$$
\begin{gathered}
Z_{n}^{m}(\rho, \phi)=R_{n}^{m}(\rho) \cos (m \phi) \Rightarrow Z_{n}^{m}(x, y)=\mathcal{R}_{n}^{m}(x, y) \theta_{m}(x, y), \\
Z_{n}^{-m}(\rho, \phi)=R_{n}^{m}(\rho) \sin (m \phi) \Rightarrow Z_{n}^{-m}(x, y)=\mathcal{R}_{n}^{m}(x, y) \theta_{-m}(x, y),
\end{gathered}
$$




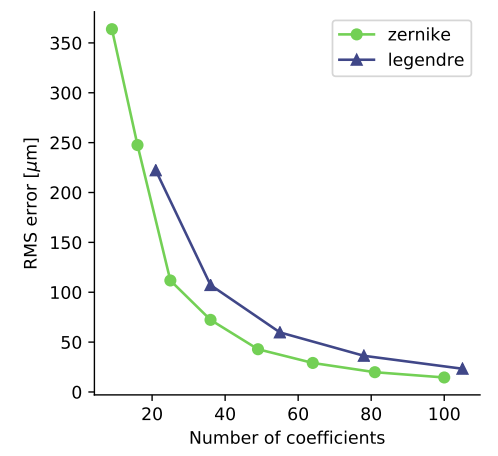

Fig. 5: RMS fit error versus number of coefficients in the fitting polynomial

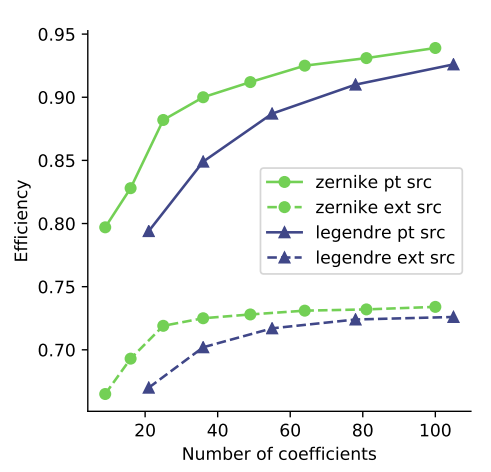

Fig. 6: Efficiency versus number of coefficients in the fitting polynomial

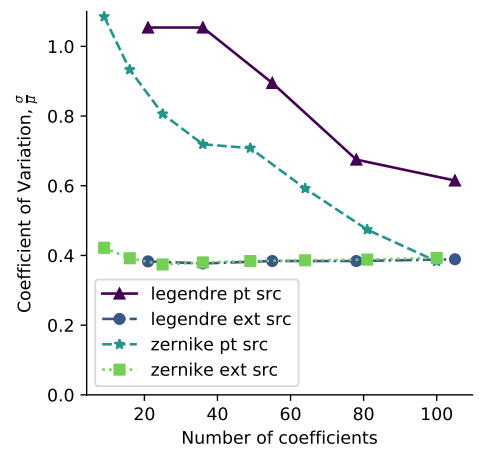

Fig. 7: Coefficient of variation across the painting area

where

$$
\begin{gathered}
\mathcal{R}_{n}^{m}=\sum_{k=0}^{n-m} \frac{(-1)^{k}(2 n-m-k)}{k !(n-k) !(n-m-k) !}\left(x^{2}+y^{2}\right)^{(n-m-k)}, \\
\theta_{m}(x, y)=\sum_{k=0}^{\frac{m}{2}}(-1)^{k}\left(\begin{array}{c}
m \\
2 k
\end{array}\right) x^{m-2 k} y^{2 k},
\end{gathered}
$$

and

$$
\theta_{-m}(x, y)=\sum_{k=0}^{\frac{m-1}{2}}(-1)^{k}\left(\begin{array}{c}
m \\
2 k+1
\end{array}\right) x^{m-2 k-1} y^{2 k+1} .
$$

The Legendre functions are built using the generating function

$$
f(x, y)=\sum_{j=1}^{\infty} a_{j} Q_{j}(x, y)
$$

where $[24]$

and the $j^{\text {th }}$ coefficient is

$$
Q_{j}(x, y)=L_{l}(x) L_{m}(y)
$$

$$
j=\frac{(l+m)^{2}+l+3 m}{2}+1
$$

with the individual polynomials described as

$$
L_{n}(k)=\sqrt{2 n+1} P_{n}(k)
$$

where

$$
\begin{aligned}
P_{0}(k) & =1, P_{1}(k)=k, \\
(n+1) P_{n+1}(k) & =(2 n+1) k P_{n}(k)-n P_{n-1}(k) .
\end{aligned}
$$

The system geometry leads to plane symmetry about the $y$-axis for the optic, allowing the odd $x$ terms to be removed. A custom MATLAB (MATLAB, Natick, MA) program builds the polynomial functions, removes the odd $x$ terms, and fits the polynomial to the point cloud.

\subsection{Create implicit surface definition files}

The MATLAB program outputs the values of the coefficients of the function, the function string, $f(x, y)$, and the derivatives of the function, $\frac{\partial f}{\partial x}, \frac{\partial f}{\partial y}$, and $\frac{\partial f}{\partial z}$. A Python (Python Software Foundation, https://www python.org) [25,26] script reads the MATLAB outputs, symbolically simplifies the function and derivative strings, and creates implicit surface definition files to be read by FRED (Photon Engineering, Tucson, AZ). 


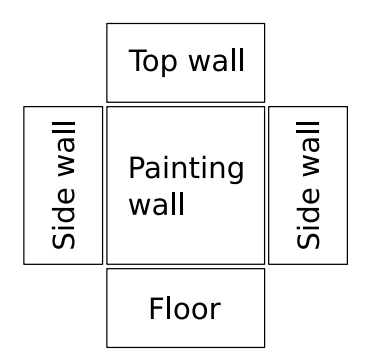

(a) Map of surfaces in Rothko exhibit

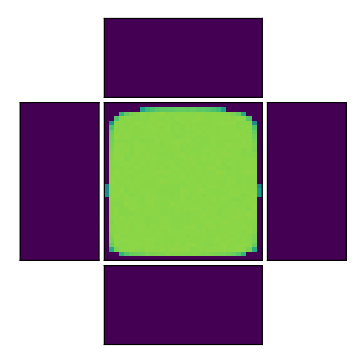

(b) STEP file, point source

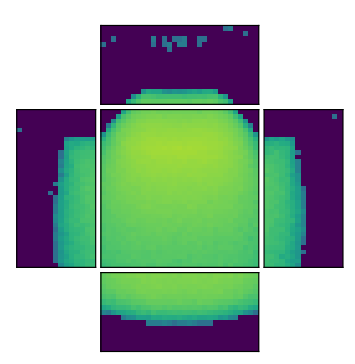

(c) STEP file, extended source

Fig. 8: Comparison of STEP representation of the initial point cloud with a Lambertian point source and a Lambertian $6 \times 6 \mathrm{~mm}$ extended source

\subsection{Trace rays and optimize}

Once the surface is properly defined, rays are traced through the surface. Symbolic simplification of the strings describing the function and derivatives is key as the ray tracing speed drastically slows as characters are added to the string. Speeds could possibly improve if the polynomial definitions were defined natively in the software package. A NURBS representation of the Cartesian oval is used to improve ray tracing speed.

\section{Results}

\subsection{Fit comparison}

Zernike fits with a square number of coefficients $(9,16,25, \ldots, 81,100)$ and Legende fits with $21,36,55$, and 78 coefficients are compared. Coefficient numbers are chosen based on the completion of a numerical order for both the $x$ and $y$ bases. An additional normalization radius in both the $x$-axis and $y$-axis is used. As shown in Figure 5, the root mean squared (RMS) error of the functional fit to the point cloud decreases as the number of coefficients is increased.

The functional surface fits are ray traced in FRED to evaluate the resulting illuminance patterns on the painting wall. To improve ray tracing speed and isolate the changes due to the functional fit, the Cartesian oval is represented as a NURBS surface generated from a STEP file of the optic. Ray tracing of a Lambertian point source and a Lambertian extended $(6 \times 6 \mathrm{~mm})$ source are compared. Figure 6 provides the efficiency [27]

$$
\eta=\frac{\Phi_{\text {target }}}{\Phi_{\text {source }}}
$$

of the optic in directing rays to the back wall. Figure 7 shows the uniformity of the illuminance across the painting area, a subset of the painting wall area. Changes in point source illumination variation and efficiency are more significant as the RMS error of the functional fit decreases whereas the extended source provides little change as additional coefficients are added.

The illuminance on the various surfaces in the room is mapped as in Figure 8a. The exact surface definition in the form of a STEP file (Figure 8) is compared to the Zernike (Figure 9) and Legendre (Figure 10) fits. All the illuminance plots are normalized to the same maximum value before calculating the logarithm of the data.

As designed, the STEP file traced with a Lambertian point source provides unity efficiency (with a limited angle source) and perfect uniformity of optical radiation to the back wall. Tracing the polynomial fits with a point source, the structure of the fitting polynomial can be readily seen in the illuminance pattern. For both the STEP file and polynomial fits traced with an extended source, the illuminance spills to the top wall, side walls and floor with an illuminance gradient between the top and bottom of the painting wall. However, the structure in the illumination pattern is reduced and overall uniformity is improved. Further optimization of the fitting function coefficients aims to decrease illuminance variation below the $25 \%$ specification and increase efficiency. 


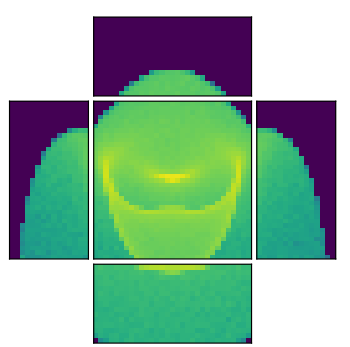

(a) Third order, point source

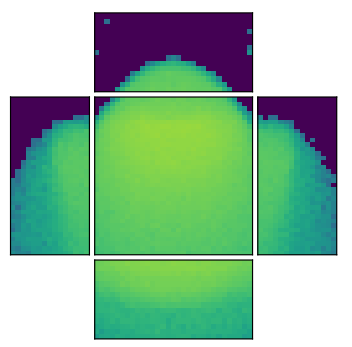

(d) Third order, extended source

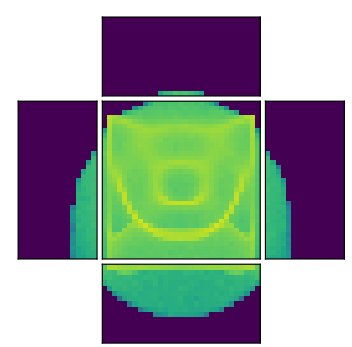

(b) Sixth order, point source

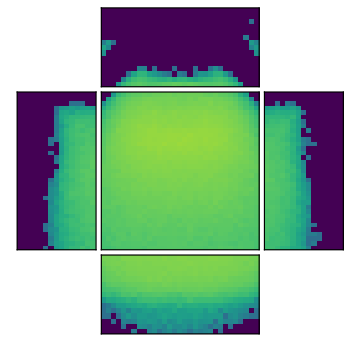

(e) Sixth order, extended source

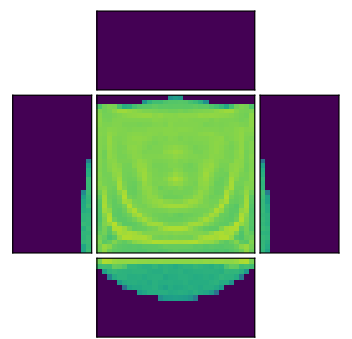

(c) Ninth order, point source
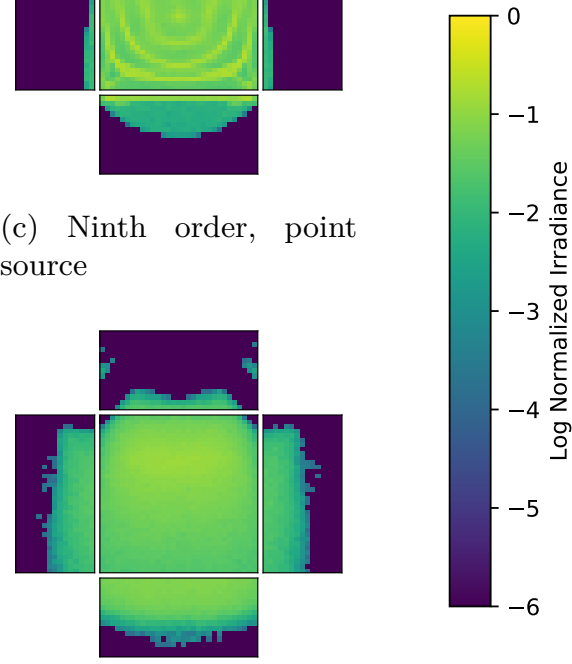

(f) Ninth order, extended source

Fig. 9: Comparison of Zernike fit orders with a Lambertian point source and a Lambertian $6 \times 6 \mathrm{~mm}$ extended source

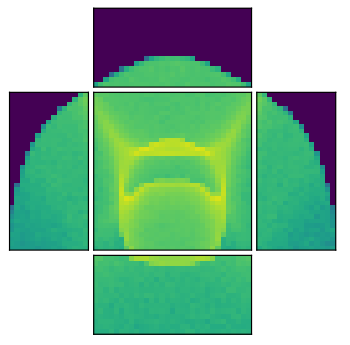

(a) Fifth order, point source

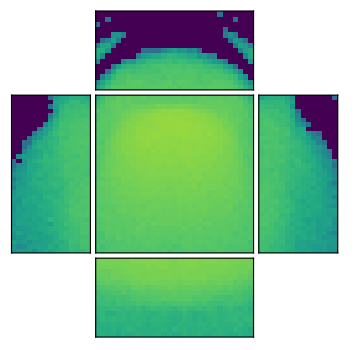

(d) Fifth order, extended source

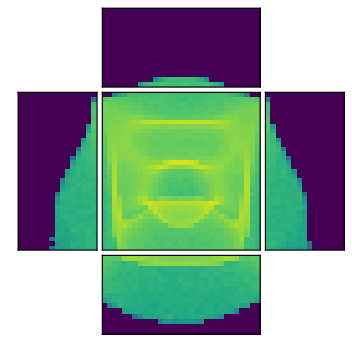

(b) Ninth order, point source

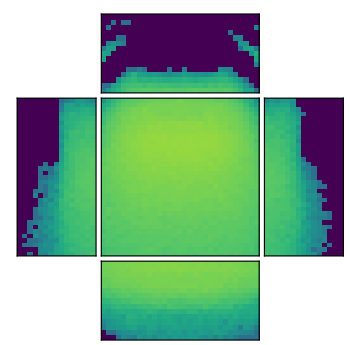

(e) Ninth order, extended source

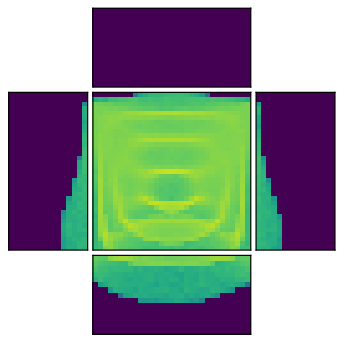

(c) Thirteenth order, point source
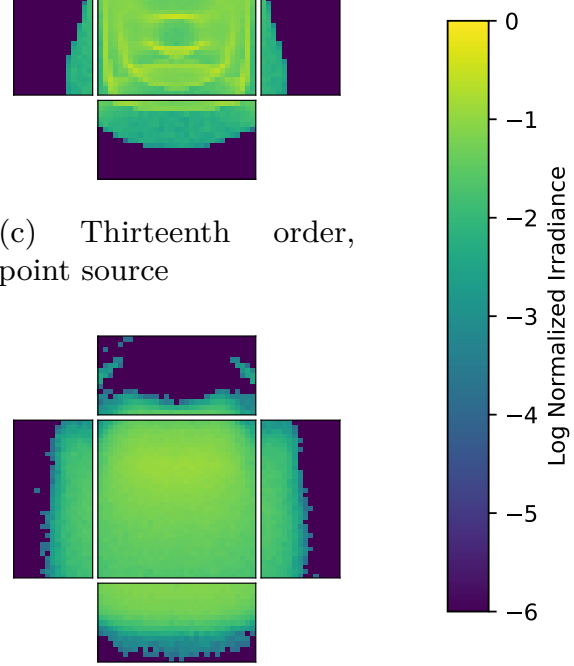

(f) Thirteenth order, extended source

Fig. 10: Comparison of Legendre fit orders with a Lambertian point source and $6 \times 6 \mathrm{~mm}$ extended source 


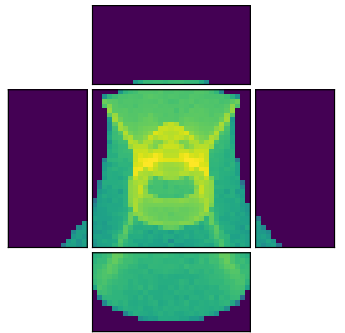

(a) Point source trace
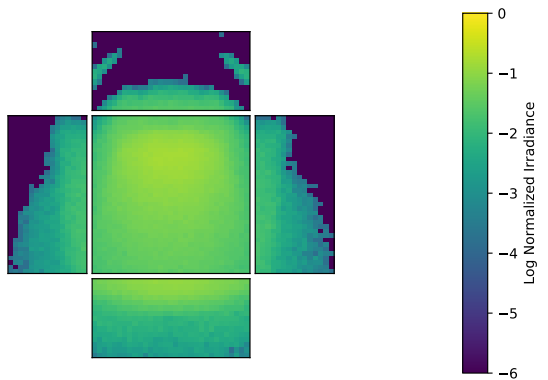

(b) Extended source trace

Fig. 11: Results for a ninth order Legendre fit optimized with an $6 \times 6 \mathrm{~mm}$ extended source

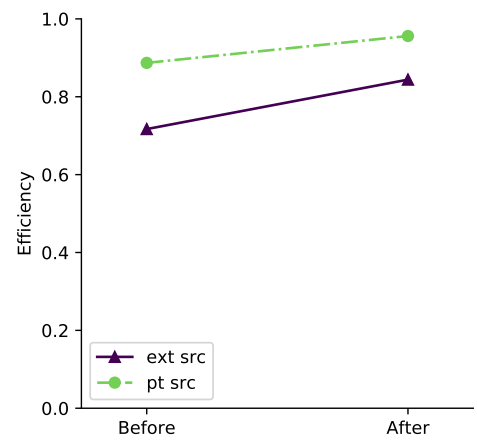

Fig. 12: Painting wall efficiency change from the nominal after optimization

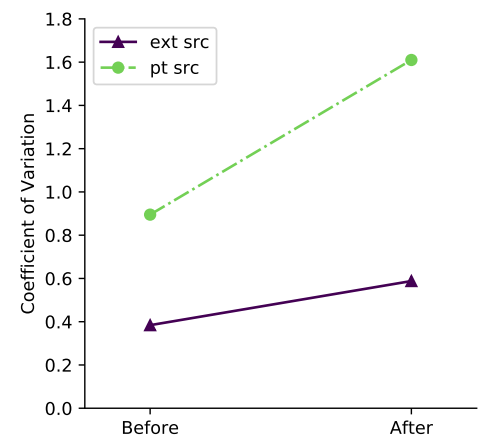

Fig. 13: Painting wall coefficient of variation change from the nominal after optimization

\subsection{Optimization results}

An optimization routine was performed on the ninth order Legendre polynomial fit with 55 coefficients. The built-in FRED optimizer was used with all polynomial coefficients and the normalization radii set as variables with limits of $\pm 10 \%$. The merit function sought to maximize the irradiance on the painting wall while minimizing the variance. The top wall's, side wall's, and floor's irradiances and variances were minimized. The optic was optimized using the extended source. The result was traced with both a point source and an extended source for comparison with the nominal. Figure 11 shows the illuminance results from each run and the change from the nominal (Figures 10b and 10e) in both traces is apparent. Figures 12 and 13 show the efficiency and uniformity change from the nominal after optimization. In this case, the optimization increased the extended source trace efficiency by $17.7 \%$ at the expense of a $53.1 \%$ increase to variation across the painting.

\section{Conclusion}

Shown is the $\mathrm{F}^{4}$ freeform optic design method applied to illuminating Mark Rothko's Green on Blue. The process and some preliminary fitting and tracing results were shown. Future work includes further refinement of the system model with addition of bi-directional reflectance distribution function (BRDF) information for the walls and floor, refinement of the optimization routine, implementation with higher order polynomial functions, and addition of assembly tolerancing metrics. Additional fitting functions will also be explored. Once a finalized design of a optic is obtained, the optic will be manufactured and a system will be built and characterized after installation at UAMA. Finally, application of the method to various illumination geometries will be explored. 


\section{Acknowledgments}

The authors would like to thank Nathan Saxton from UAMA for his discussions and initiating the collaboration between UAMA and College of Optical Sciences and to Jessica Steidle for her contributions during her REU. We are grateful for our funding sources. Ross is funded by the NIH Biomedical Imaging and Spectroscopy Training Grant T32EB000809 and the SPIE Optical Design and Engineering Scholarship. Rachel is funded by the College of Optical Sciences. Kaitlyn is funded by the Friends of Tucson Optics (FOTO) Boye Family Graduate Student Scholarship in Optical Sciences and the SPIE and Photonics Media Teddi Laurin Scholarship.

\section{References}

1. Bloomberg, "Billionaire paid $\$ 500$ million for abstract expressionist paintings in record art deal," The Telegragh (Feb. 2016).

2. Harvard University. Art Museums. Center for Conservation and Technical Studies, [Mark Rothko's Harvard Murals], Center for Conservation and Technical Studies, Harvard University Art Museums, Cambridge, MA (1988).

3. Ma, D., Pacheco, S., Wang, C., and Liang, R., "Freeform optics construction with nonuniformly sampled grids in modified double-pole coordinate system," Opt. Eng. 54(12), 125102-125102 (2015).

4. Feng, Z., Froese, B. D., and Liang, R., "Freeform illumination optics construction following an optimal transport map," Appl. Opt. 55, 4301 (June 2016).

5. Fournier, F. R., Cassarly, W. J., and Rolland, J., "Fast freeform reflector generation using source-target maps," Opt. Express 18, 5295-5304 (Mar. 2010).

6. Minano, J. C., Benitez, P., Cvetkovic, A., and Mohedano, R., "SMS 3D design method," in [Illumination Engineering: Design with Nonimaging Optics], Koshel, R. J., ed., 101-146, IEEE Press, John Wiley \& Sons, Inc., Hoboken, N.J (2013).

7. Wu, R., Hua, H., Benítez, P., Miñano, J. C., and Liang, R., "Design of compact and ultra efficient aspherical lenses for extended Lambertian sources in two-dimensional geometry," Opt. Express 24, 5078 (Mar. 2016).

8. Wu, R., Huang, C. Y., Zhu, X., Cheng, H.-N., and Liang, R., "Direct three-dimensional design of compact and ultra-efficient freeform lenses for extended light sources," Optica 3, 840 (Aug. 2016).

9. Stenger, J., Khandekar, N., Wilker, A., Kallsen, K., Kirby, D. P., and Eremin, K., "The making of Mark Rothko's Harvard Murals," Stud. Conserv. 61, 331-347 (Nov. 2016).

10. Stenger, J., Khandekar, N., Raskar, R., Cuellar, S., Mohan, A., and Gschwind, R., "Conservation of a room: A treatment proposal for Mark Rothko's Harvard Murals," Stud. Conserv. 61, 348-361 (Nov. 2016).

11. Illuminating Engineering Society of North America, "Lighting for Public Places and Institutions," in [The IESNA Lighting Handbook: Reference and Application], Rea, M. S., ed., Illuminating Engineering Society of North America, New York, NY, 9th ed. (2000).

12. International Commission on Illumination, "Technical Report 157:2004: Control of Damage to Museum Objects by Optical Radiation," (2004).

13. Rothko, C., "How Not to Hang a Rothko," Unpublished essay (2015).

14. Rothko, M., ""Letter to Katherine Kuh", September 25, 1954," in [Writings on Art], Lopez-Remiro, M., ed., 99, Yale University Press, New Haven (2007).

15. Druzik, J. R. and Michalski, S., [Guidelines for Selecting Solid-State Lighting for Museums], Getty Conservation Institute (2012).

16. Miller, N. J. and Druzik, J. R., "Demonstration Assessment of Light-Emitting Diode (LED) Retrofit Lamps at an Exhibit of 19th Century Photography at the Getty Museum," tech. rep., Pacific Northwest National Lab.(PNNL), Richland, WA (United States) (2012).

17. Miller, N. J. and Rosenfeld, S. M., "Demonstration of LED retrofit lamps at the Smithsonian American Art museum, Washington, DC," tech. rep., Pacific Northwest National Laboratory (PNNL), Richland, WA (US) (2012).

18. Kahn, A. M., "LED Freeform Lens Design for Rectangular Illumination in Museums," opti485 design review, College of Optical Sciences, The University of Arizona (May 2015). 
19. Everett-Haynes, L. M., "A Light Touch for Museum Artwork," UANews (May 2015).

20. Fechner, G. T., [Elements of Psychophysics], Holt, Reinhart, and Winston, New York (1966).

21. Gross, H., Broemel, A., Beier, M., Steinkopf, R., Hartung, J., Zhong, Y., Oleszko, M., and Ochse, D., "Overview on surface representations for freeform surfaces," in [Proc. of SPIE: Optical Design and Engineering], Mazuray, L., Wartmann, R., and Wood, A. P., eds., 9626, 96260U (Sept. 2015).

22. Mahajan, V. N., [Optical Imaging and Aberrations, Part III: Wavefront Analysis], SPIE Optical Engineering Press, Bellingham, Wash (2013).

23. Williams, K. E., Parameterizing Freeform Optical Surfaces for the Optimized Design of Imaging and Illumination Systems, master's thesis, The University of Arizona, Tucson, AZ (2017).

24. Arfken, G. B. and Weber, H.-J., [Mathematical Methods for Physicists], Elsevier, Boston, 6th ed ed. (2005).

25. Hunter, J. D., "Matplotlib: A 2D Graphics Environment," Computing in Science 8$\}$ Engineering 9(3), 90-95 (May-June 2007).

26. Meurer, A., Smith, C. P., Paprocki, M., Čertík, O., Kirpichev, S. B., Rocklin, M., Kumar, A., Ivanov, S., Moore, J. K., Singh, S., Rathnayake, T., Vig, S., Granger, B. E., Muller, R. P., Bonazzi, F., Gupta, H., Vats, S., Johansson, F., Pedregosa, F., Curry, M. J., Terrel, A. R., Roučka, Š., Saboo, A., Fernando, I., Kulal, S., Cimrman, R., and Scopatz, A., "SymPy: Symbolic computing in Python," PeerJ Comput. Sci. 3, e103 (Jan. 2017).

27. Koshel, R. J., [Illumination Engineering: Design with Nonimaging Optics], IEEE Press ; Wiley, Piscataway, NJ : Hoboken, New Jersey (2013). 